\title{
Prognostic Impact of $\beta 2$ Adrenergic Receptor Expression in Surgically Resected Pulmonary Pleomorphic Carcinoma
}

\author{
KYOICHI KAIRA ${ }^{1,2}$, MITSUHIRO KAMIYOSHIHARA ${ }^{3}$, OSAMU KAWASHIMA ${ }^{4}$, \\ HIDEKI ENDOH $^{5}$, KAZUYOSHI IMAIZUMI ${ }^{6}$, MASAYUKI SUGANO ${ }^{7}$, \\ SHIGEFUMI TANAKA ${ }^{8}$, ATSUSHI FUJITA ${ }^{9}$, YOSHIHITO KOGURE ${ }^{10}$, AKIRA SHIMIZU ${ }^{11}$, \\ TETSUNARI OYAMA ${ }^{12}$, TAKAYUKI ASAO ${ }^{13}$, KIMIHIRO SHIMIZU ${ }^{14^{*}}$ and AKIRA MOGI ${ }^{1 * *}$ \\ ${ }^{1}$ Department of Oncology Clinical Development, Gunma University \\ Graduate School of Medicine, Maebashi, Japan; \\ ${ }^{2}$ Department of Respiratory Medicine, Comprehensive Cancer Center, International \\ Medical Center, Saitama Medical University, Hidaka, Japan; \\ ${ }^{3}$ Department of Respiratory Surgery, Maebashi Red Cross Hospital, Maebashi, Japan; \\ ${ }^{4}$ Department of Respiratory Surgery, Shibukawa Medical Center, Shibukawa, Japan; \\ ${ }^{5}$ Department of Thoracic Surgery, Saku Central Hospital Advanced Care Center, Saku, Japan; \\ ${ }^{6}$ Department of Respiratory Medicine, Fujita Health University, Toyoake, Japan; \\ ${ }^{7}$ Department of Respiratory Surgery, Takasaki Medical Center, Takasaki, Japan; \\ ${ }^{8}$ Department of Respiratory Surgery, Isesaki Municipal Hospital, Isesaki, Japan; \\ ${ }^{9}$ Division of Respiratory Surgery, Gunma Prefectural Cancer Center, Ota, Japan; \\ ${ }^{10}$ Department of Respiratory Medicine, Nagoya Medical Center, Nagoya, Japan; \\ ${ }^{11}$ Department of Dermatology, Gunma University Graduate School of Medicine, Maebashi, Japan; \\ ${ }^{12}$ Department of Diagnostic Pathology, Gunma University Graduate School of Medicine, Maebashi, Japan; \\ ${ }^{13}$ Big Data Center for Integrative Analysis, Gunma University Initiative for Advance Research, Maebashi, Japan; \\ ${ }^{14}$ Division of Respiratory Surgery, Integrative Center of General \\ Surgery, Gunma University Hospital, Maebashi, Japan
}

\begin{abstract}
Background/Aim: The $\beta 2$-adrenergic receptor $(\beta 2 A R)$ is highly expressed in various human cancers and has been linked to tumor growth and metastases. Although $\beta 2 A R$ is considered a novel therapeutic target of human neoplasms, the clinicopathological significance of $\beta 2 A R$ expression in patients with pulmonary pleomorphic carcinoma (PPC) remains unclear. The aim of this study was to clarify the prognostic impact of $\beta 2 A R$ in PPC. Patients and Methods: One hundred and five Japanese patients with surgically
\end{abstract}

*These Authors contributed equally to this study.

Correspondence to: Kyoichi Kaira, Department of Oncology Clinical Development, Gunma University Graduate School of Medicine, 3-39-15, Showa-machi, Maebashi, Gunma 371-8511, Japan; Department of Respiratory Medicine, Comprehensive Cancer Center, International Medical Center, Saitama Medical University, 1397-1 Yamane, Hidaka-City, Saitama, 350-1298, Japan. Tel: +81 272208122, Fax: +81 272020212, e-mail: kkaira1970@yahoo.co.jp

Key Words: $\beta 2$ Adrenergic receptor, pulmonary pleomorphic carcinoma, immunohistochemistry, prognosis. resected $P P C$ were included in the study. The expression levels of $\beta 2 A R$ were assessed by immunohistochemistry in specimens from the resected tumors, and their association with patient survival, as well as with tumor characteristics was investigated. Results: $\beta 2 A R$ was highly expressed in $63 \%$ of all patients, irrespective of adenocarcinoma components present. The $\beta 2 A R$ expression was significantly associated with lymph node metastasis, lymphatic permeation and tumor cell proliferation in PPC patients with early-stage disease (stage I or II). A high $\beta 2 A R$ expression was identified as a significant predictor of worse prognosis for PPC patients during early stages of the disease. Multivariate analysis confirmed that $\beta 2 A R$ expression was an independent factor for predicting the overall survival of PPC patients. Conclusion: $\beta 2 A R$ can serve as a significant predictor of tumor aggressiveness and poor survival for PPC patients, especially those with early-stage disease.

Pulmonary pleomorphic carcinoma (PPC) is a rare tumor characterized by aggressiveness and chemotherapeutic resistance, with an incidence ranging from $0.1-0.4 \%$ of all lung cancers (1). PPC includes carcinomatous and sarcomatoid 
components, and is classified as a subtype of sarcomatoid carcinoma of the lung in the World Health Organization histological classification of lung neoplasms (2). Because of its rarity, no established biomarker has yet been linked to the prognosis or therapeutic response of patients with PPC. Furthermore, the clinicopathological features of PPC have not been fully clarified. Further investigation is needed to discover the molecular mechanisms related to the identification of possible biomarkers for PPC.

Beta-blockers are often administered for the treatment of heart disease (3). The $\beta 2$-adrenergic receptor $(\beta 2-A R)$, a member of the transmembrane $G$ protein-coupled receptor (GPCR) family, triggers multiple signaling cascades and regulates cell proliferation through a classical cyclicadenosine-monophosphate (cAMP)/protein kinase A (PKA) pathway (4). Chemokines and neurotransmitters (ligands) bind to GPCRs and play essential roles in regulating cancer recurrence (5). A study in a murine ovarian cancer model demonstrated that beta-adrenergic activation of the cAMPPKA signaling pathway via $\beta 2$-AR affects angiogenesis, followed by tumor proliferation and cell growth (6).

$\beta 2-A R$ is highly expressed in various human neoplasms, such as breast cancer, oral cancer, melanoma, prostate cancer, gastric cancer, lung cancer and hepatocellular carcinoma, while overexpression of $\beta 2$-AR is closely linked to negative prognosis, tumor recurrence, and metastases (714). Recent reports have described a significant reduction of tumor recurrence in breast cancer patients taking betablockers compared to those not prescribed this type of drug $(15,16)$. In addition, beta-blockers, especially propranolol, appear to prolong the survival of patients with breast cancer (16). Two preclinical studies in oral squamous cell carcinoma (SQC) and breast cancer have documented that $\beta$-adrenergic signaling is associated with: i) the inhibition of apoptosis, ii) the induction of vascular endothelial growth factor (VEGF) expression, and iii) the occurrence of metastases $(9,16)$. Yazawa et al. reported that $\beta 2-\mathrm{AR}$ is highly expressed in approximately $30 \%$ of all patients with non-small cell lung cancer (NSCLC); significantly correlating with lymphatic permeation, vascular invasion, and tumor cell proliferation (13). All this evidence could constitute $\beta 2-\mathrm{AR}$ as an independent prognostic factor for early-stage pulmonary adenocarcinoma (AC) (12); however, the clinicopathological significance of $\beta 2-\mathrm{AR}$ expression in patients with PPC remains unclear.

In this context, the present study assessed the prognostic significance of $\beta 2$-AR expression in patients with completely resected PPC.

\section{Patients and Methods}

Patients. Between August 2001 and October 2015, 105 Japanese patients with histologically confirmed PPC who underwent surgical resection at multiple institutions were enrolled in this study. Pleomorphic carcinoma was diagnosed according to the 2015 World Health Organization Classification of Tumours (2). Diagnoses were confirmed by light microscopy and immunohistochemistry. PPC was defined as NSCLC containing at least $10 \%$ sarcomatoid components. This study included 105 surgically resected primary tumors in accordance with institutional guidelines of the Gunma University Hospital and the Declaration of Helsinki. The institutional review boards of all participating institutions approved this study. In the present study, mortality and recurrence were determined using medical records. The currently collected tumor samples used in this study were performed using those of our previous study (17).

Immunohistochemical staining. $\beta 2 \mathrm{AR}$ expression was assessed by immunohistochemical staining using a rabbit anti-human $\beta 2 \mathrm{AR}$ monoclonal antibody (Abcam, Inc., Cambridge, UK; 1:100 dilution) against a C-terminal peptide of the human $\beta 2 \mathrm{AR}$ protein. The reaction was visualized using the Histofine Simple Stain MAX-PO (Multi) Kit (Nichirei, Tokyo, Japan) according to the manufacturer's instructions. The detailed protocol for immunostaining has been published elsewhere (13-15). Negative controls were incubated without primary antibody and no detectable staining was evident. 32AR expression was considered positive only if distinct cytoplasmic and plasma membrane staining was present. $\beta 2 \mathrm{AR}$ expression was scored as follows: i) score $1, \leq 10 \%$ of tumor area stained; ii) score 2, 11-25\% stained; score 3, 26-50\% stained; and score $4, \geq 51 \%$ stained. Tumors in which the stained tumor cells scored $\geq 4$ were considered as "high-expression" tumors.

Immunohistochemical staining for $\mathrm{Ki}-67$ was performed as described previously (12) using a murine monoclonal antibody against Ki-67 (Dako, Glostrup, Denmark; 1:40 dilution). Areas of high cell density in the immunostained sections were assessed for Ki-67 expression. All epithelial cells with nuclear staining at any intensity were defined as positive. Approximately 1,000 nuclei were counted on each slide. Proliferative activity was assessed as a percentage of $\mathrm{Ki}-67$-stained nuclei (Ki-67 labeling index) in the sample. Tumor cells with a Ki-67 labeling index value greater than the calculated median value were defined as strong positive. All sections were assessed using light microscopy in a blinded fashion by at least two investigators. In case of discrepancies, all parties evaluated the slides simultaneously until reaching a final consensus. None of the investigators had knowledge of the patients' outcomes.

Statistical analysis. Statistical analyses were performed using Student's $t$ - and $\chi^{2}$-tests for continuous and categorical variables, respectively. Correlations were analyzed using nonparametric Spearman's rank tests. The Kaplan-Meier method was used to estimate survival as a function of time and survival differences were analyzed by log-rank tests. Overall survival (OS) was the time from tumor resection to death due to any cause. Disease-free survival (DFS) was the time between tumor resection and the first episode of disease progression or death. Univariate and multivariate survival analyses were performed using the Cox proportional hazards model and a logistic regression model for radical surgery. All $p$-values $<0.05$ were considered statistically significant. All statistical analyses were performed using GraphPad Prism version 4 (GraphPad Software, San Diego, CA, USA) and JMP Pro version 12.0 (SAS Institute Inc., Cary, NC, USA). 

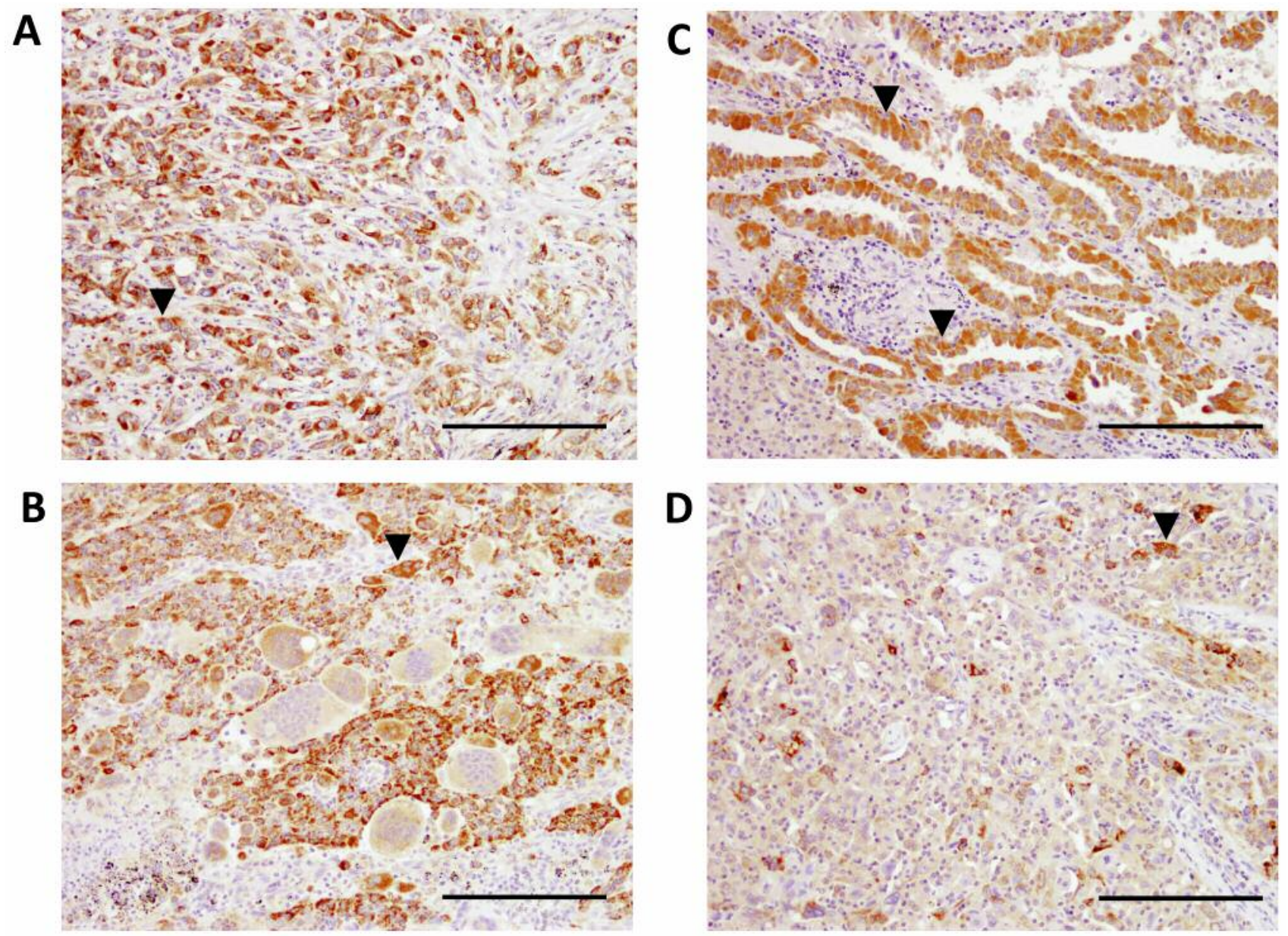

Figure 1. Immunohistochemical staining of $\beta 2$-adrenergic receptor $(\beta 2 A R)$ in pulmonary pleomorphic carcinoma. Positive staining is evident in the cytoplasm and in plasma membranes of cancer cells. Figures $A, B$ and $C$ are representative of score $=4$ and $D$ of score $=2$. The $\beta 2 A R-p o s i t i v e$ immunostaining was observed in the sarcomatous components of spindle cells $(A)$, giant cells $(B)$ and epithelial components of the adenocarcinoma (C). (magnification 200x, scale bar $200 \mu$ m; black arrowheads show $\beta 2 A R$-positive cells).

\section{Results}

Patient characteristics. A total of 105 patients (79 men, 26 women; median age 69 years, range $=35-88$ years) were analyzed in the current study. Thirty-four patients $(32 \%)$ were diagnosed with stage I, 37 (35\%) with stage II, 27 (26\%) with stage III, and seven (7\%) with stage IV PPC. Eighty-four patients $(80 \%)$ had a smoking history. The breakdown of sarcomatous components included spindle cell type (69 patients), giant cell type (10 patients), both spindle cell and giant cell type (13 patients), and other types (13 patients). Histological analysis revealed 29 patients with a mix of carcinomatous and sarcomatous components. The carcinomatous components of 76 patients included 48

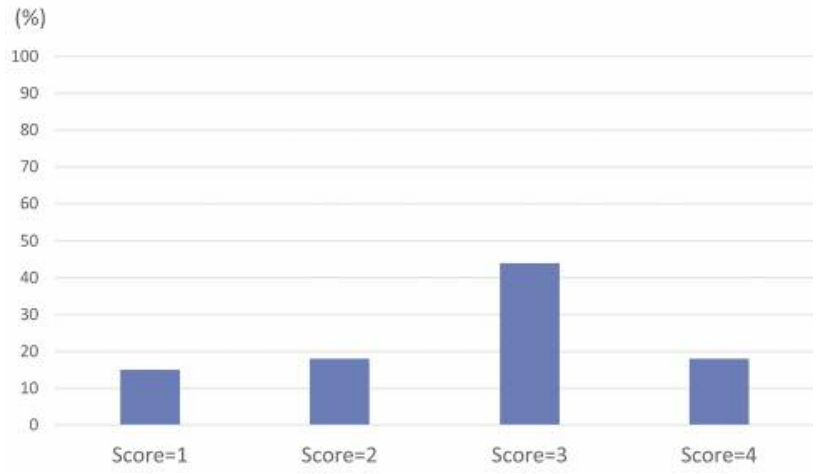

Figure 2. The percentages of tumor specimens with $\beta 2$-adrenergic receptor $(\beta 2 A R)$ expression according to scoring of 1 to 4 . 
Table I. Patient's demographics according to $\beta 2-A R$ expression.

\begin{tabular}{|c|c|c|c|c|c|c|c|c|}
\hline \multirow[t]{2}{*}{ Variables } & \multicolumn{4}{|c|}{$\beta 2-\mathrm{AR}$ Expression in all patients } & \multicolumn{4}{|c|}{$\beta 2$-AR Expression in patients with early stage } \\
\hline & $\begin{array}{c}\text { Total } \\
(\mathrm{n}=105)\end{array}$ & $\begin{array}{l}\text { High } \\
(n=66)\end{array}$ & $\begin{array}{l}\text { Low } \\
(\mathrm{n}=39)\end{array}$ & $p$-Value & $\begin{array}{c}\text { Total } \\
(\mathrm{n}=69)\end{array}$ & $\begin{array}{l}\text { High } \\
(n=43)\end{array}$ & $\begin{array}{l}\text { Low } \\
(\mathrm{n}=26)\end{array}$ & $p$-Value \\
\hline \multicolumn{9}{|l|}{ Age } \\
\hline$<69$ years $/ \geq 69$ years & $54 / 51$ & $33 / 33$ & $21 / 18$ & 0.84 & $35 / 34$ & $20 / 23$ & $15 / 11$ & 0.45 \\
\hline \multicolumn{9}{|l|}{ Gender } \\
\hline Male/Female & $79 / 26$ & $48 / 18$ & $29 / 10$ & $>0.99$ & $55 / 14$ & $34 / 9$ & $21 / 5$ & $>0.99$ \\
\hline \multicolumn{9}{|l|}{ Smoking } \\
\hline Yes/No & $84 / 21$ & $50 / 16$ & $34 / 5$ & 0.21 & $59 / 10$ & $35 / 8$ & $24 / 2$ & 0.29 \\
\hline \multicolumn{9}{|l|}{ Tumor factor } \\
\hline $\mathrm{T} 1-2 / \mathrm{T} 3-4$ & $65 / 40$ & $38 / 28$ & $27 / 12$ & 0.29 & $53 / 16$ & $31 / 12$ & $22 / 4$ & 0.37 \\
\hline \multicolumn{9}{|l|}{ Node factor } \\
\hline Absent/Present & $72 / 33$ & $48 / 18$ & $24 / 15$ & 0.38 & $50 / 9$ & $38 / 5$ & $12 / 14$ & $<0.01 *$ \\
\hline \multicolumn{9}{|l|}{ Stage } \\
\hline I-II/III-IV & $69 / 36$ & $43 / 23$ & $26 / 13$ & $>0.99$ & - & - & - & - \\
\hline \multicolumn{9}{|l|}{ Lymphatic permeation } \\
\hline Absent/Present & $41 / 64$ & $23 / 43$ & $18 / 21$ & 0.30 & $35 / 34$ & $17 / 26$ & $18 / 8$ & $0.03 *$ \\
\hline \multicolumn{9}{|l|}{ Vascular invasion } \\
\hline Absent/Present & $31 / 74$ & $19 / 47$ & $12 / 27$ & 0.82 & $20 / 49$ & $11 / 32$ & $9 / 17$ & 0.42 \\
\hline \multicolumn{9}{|l|}{ Pleural invasion } \\
\hline Absent/Present & $49 / 56$ & $29 / 37$ & $20 / 19$ & 0.54 & $34 / 35$ & $18 / 25$ & $16 / 10$ & 0.14 \\
\hline \multicolumn{9}{|l|}{ Adjuvant chemotherapy } \\
\hline Absent /Present & $78 / 27$ & $46 / 20$ & $32 / 7$ & 0.17 & $50 / 19$ & $30 / 13$ & $20 / 6$ & 0.58 \\
\hline \multicolumn{9}{|l|}{ Ki-67 labeling index } \\
\hline High/Low & $50 / 55$ & $37 / 29$ & $13 / 26$ & $0.03^{*}$ & $34 / 35$ & $26 / 17$ & $8 / 18$ & $0.02 *$ \\
\hline
\end{tabular}

$* p<0.05$ is considered statistically significant. $t$-Test score for continuous variables and $\chi^{2}$ test for categorical variables. $\beta 2-\mathrm{AR}$, Beta-2 adrenergic receptor.

adenocarcinomas, 13 squamous cell carcinomas, 8 adenosquamous cell carcinomas, 2 poorly differentiated carcinomas, and 5 large cell carcinomas. Postoperative survival was measured from the day of the surgery. The median follow-up period was 476 days (range $=30-4,519$ days).

$\beta 2 A R$ expression and clinical correlation. All primary sites with PPC were analyzed using immunohistochemistry. Figure 1 shows representative images of $\beta 2 \mathrm{AR}$ expression in PPC patient samples. $\beta 2 \mathrm{AR}$ immunostaining was detected predominantly in the cytoplasm and in the plasma membrane of melanoma cells. The rates of high expression and average scores (from 1 to 4 ) for $\beta 2 A R$ were $63 \%(66 / 105)$ and 2.6 \pm 0.9 , respectively. The frequency of $\beta 2 \mathrm{AR}$ expression with a score of 3 was significantly higher than that of scores 1,2 or 4 (Figure 2). Concerning the epithelial phenotype, the percentages of high $\beta 2 \mathrm{AR}$ expression in patients with $\mathrm{AC}$ and non-AC components were at $63 \%$ for both $(30 / 48$ and $36 / 57$, respectively), with no statistical difference between the two groups. The average Ki-67 labeling index was 30 $21 \%$ (median, 31\%), ranging from $1-89 \%$ in all patients. A high Ki67 labeling index was observed in 47\% (50/105) of cases.

Table I shows the patients' characteristics according to $\beta 2 \mathrm{AR}$ expression. There was a statistically significant correlation between $\beta 2 \mathrm{AR}$ expression and tumor cell proliferation according to $\mathrm{Ki}-67$ labeling index in all patients (Spearman's rank; r=0.21, $p=0.03$ ). High expression of $\beta 2 \mathrm{AR}$ was also closely linked to lymph node metastasis, lymphatic permeation and tumor cell proliferation in patients with early stage disease (Spearman's rank; $r=0.23, p=0.04$ ).

Figure 3. Kaplan-Meier survival curves of overall survival (OS) and disease-free survival (DFS) according to the $\beta 2$-adrenergic receptor $(\beta 2 A R)$ expression levels. In all patients, a statistically significant difference in $O S$ was observed between patients with high and low $\beta 2 A R$ expression $(p<0.01)(A)$, whereas there was no statistically significant difference in DFS ( $p=0.25)(B)$. Statistically significant differences in both $O S(p<0.01)(C)$ and DFS ( $p=0.04)(D)$ were evident between early disease (stage I or II) patients with high and low $\beta 2 A R$ expression, but not in patients with advanced disease (stage III or IV) (Figure E; OS, $p=0.41$, and Figure F; DFS, $p=0.64$ ). Kaplan-Meier survival curves according to the type of epithelial phenotype (adenocarcinoma or non-adenocarcinoma). A statistically significant difference in $O S$ between high and low $\beta 2 A R$ expression groups was observed in patients with non-adenocarcinoma component $(p=0.02)(G)$, but not in those with adenocarcinoma component $(p=0.14)(H)$. 

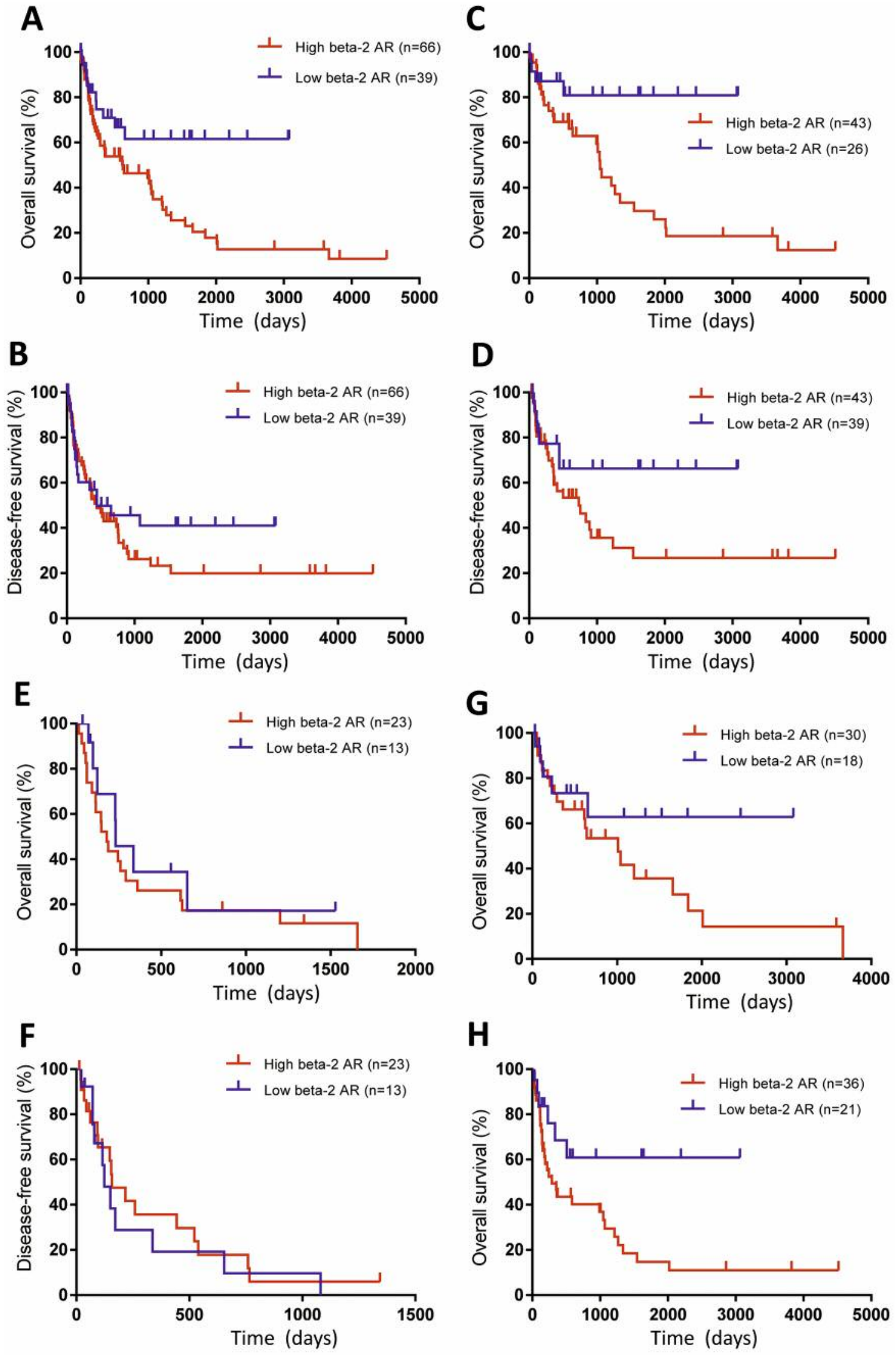
Survival analysis. The median DFS and OS rates for all patients were 443 days and 991 days, respectively; while for patients with $\mathrm{AC}$ were 522 days and 1,038 days, and for those with non-AC components were 336 days and 507 days, respectively. Of the 105 patients, 60 died and 45 developed recurrence after initial surgery. Table II shows the univariate and multivariate analyses of all patients and of those with early stage disease. Figure 3 shows the Kaplan-Meier survival curve for patients with high or low $\beta 2$-AR expression. In all patients, univariate analysis revealed that disease stage and $\beta 2 \mathrm{AR}$ expression were significant prognostic factors for predicting OS, while disease stage, lymphatic permeation, and pleural involvement were closely linked to worse DFS. Based on the results of the univariate log-rank test, we screened variables with a cut-off of $p<0.05$. In the multivariate analysis, the disease stage and $\beta 2 \mathrm{AR}$ expression were both identified as independent prognostic markers for predicting OS, while disease stage and pleural involvement were significant prognostic markers for DFS.

Survival analysis according to disease stage revealed that a high expression of $\beta 2 \mathrm{AR}$ was closely linked to a worse prognostic prediction for patients with early stage disease (Figure 3A), but not for those with advanced disease (Figure $3 B)$. In the univariate analysis of patients with early stage disease, vascular invasion and $\beta 2 \mathrm{AR}$ expression were significant predictors of OS, while vascular invasion, pleural involvement, and $\beta 2 \mathrm{AR}$ expression were associated with worse DFS. Multivariate analysis showed that vascular invasion and $\beta 2 \mathrm{AR}$ expression were independent prognostic factors for predicting poor OS in patients with early-stage disease; while no significant predictors were identified for DFS.

Next, we performed a survival analysis according to the type of epithelial phenotypes (AC or non-AC). A statistically significant difference in OS was observed between high and low $\beta 2 \mathrm{AR}$ expression in patients with non-AC components (Figure 3C), but not in those with AC components (Figure $3 \mathrm{D})$. Figure 4 shows the forest plot of one-year OS and DFS rates, according to the $\beta 2 \mathrm{AR}$ expression levels for each variable. Patients with high $\beta 2 A R$ expression exhibited a shorter OS compared to those expressing low levels of $\beta 2 \mathrm{AR}$, particularly if they are female patients, or during early-stage disease (I or II), in the presence of positive lymphatic permeation and vascular invasion, and when there is lack of pleural involvement. DFS was significantly worse for patients with high levels of $\beta 2 \mathrm{AR}$ expression compared to those with low expression, particularly if in early-stage disease, and in the absence of vascular invasion and pleural involvement.

\section{Discussion}

This clinicopathological study assessed the prognostic impact of $\beta 2 \mathrm{AR}$ expression in patients with surgically resected PPC. We found that $\beta 2 \mathrm{AR}$ is highly expressed in patients with PPC and that this phenotype can be useful as an independent prognostic marker to predict a negative outcome after surgery, especially in PPC patients with early stage disease. A high expression of $\beta 2 \mathrm{AR}$ was closely related to lymph node metastasis, lymphatic permeation, and tumor cell proliferation in these patients. Our results suggest that the expression of $\beta 2 \mathrm{AR}$ plays an essential role in tumor progression and survival of PPC patients with early stage disease. PPC is considered a rare and dismal malignancy, and promising targets for this pathology are still lacking. $\beta 2 \mathrm{AR}$ may be a molecular target to suppress tumor growth and metastases in PPC patients.

In our study, $\beta 2$ AR was highly expressed in $63 \%$ of all PPC patients and its expression did not differ significantly between patients with $\mathrm{AC}$ and non- $\mathrm{AC}$ components. In patients with resected NSCLC, $\beta 2 \mathrm{AR}$ was highly expressed in $27 \%$ of all patients, $29 \%$ of AC patients, and $24 \%$ of non$\mathrm{AC}$ patients (12). The frequency of high $\beta 2 \mathrm{AR}$ expression was higher in patients with PPC compared to those with NSCLC. Regardless of histological type, the expression of $\beta 2 \mathrm{AR}$ was high within cancer cells. We have previously reported the frequency of high $\beta 2 \mathrm{AR}$ expression in gastric cancer and in melanoma using similar methods as here; the rates of high $\beta 2 \mathrm{AR}$ expression in gastric cancer and malignant melanoma were $30.2 \%$ and $44.4 \%$, respectively (13-14). In these studies, the expression of $\beta 2 \mathrm{AR}$ was associated with lymph node metastasis and poorer survival in PPC patients (13-14).

An increased $\beta 2 A R$ expression is observed in different human neoplasms, but its prognostic impact differs depending on the histological type of each cancer. High expression levels of $\beta 2 \mathrm{AR}$ are linked to better survival in oral SQC (18), but have a worse outcome for patients with non-small cell lung cancer, especially those with AC (12). It remains unclear why the prognostic significance of $\beta 2 \mathrm{AR}$ differs according to histological type. In patients with lung cancer, including PPC, there is a significant relationship between increased $\beta 2 \mathrm{AR}$ expression and poorer survival, regardless of histological type (12). Taken together, our study shows a positive correlation between $\beta 2 \mathrm{AR}$ and tumor aggressiveness and raises interesting questions about the role of $\beta 2 \mathrm{AR}$ in tumor progression and metastasis in PPC patients with early stage disease (stage I or II). It would be interesting to see if inhibition of $\beta 2 \mathrm{AR}$ could suppress tumor progression and metastasis in these patients

In experimental studies, propranolol, a $\beta 2 \mathrm{AR}$ antagonist, reportedly diminishes the norepinephrine-induced stimulation of cancer cell proliferation and inhibits cancer cell growth $(19,20)$. The inhibition of $\beta 2$-adrenergic signaling also contributes to suppressed tumor progression, metastasis, and recurrence in breast cancer $(15,16)$, while the inhibition of $\beta 2 \mathrm{AR}$ by propranolol offers a clinical potential as a chemotherapeutic agent for malignant 


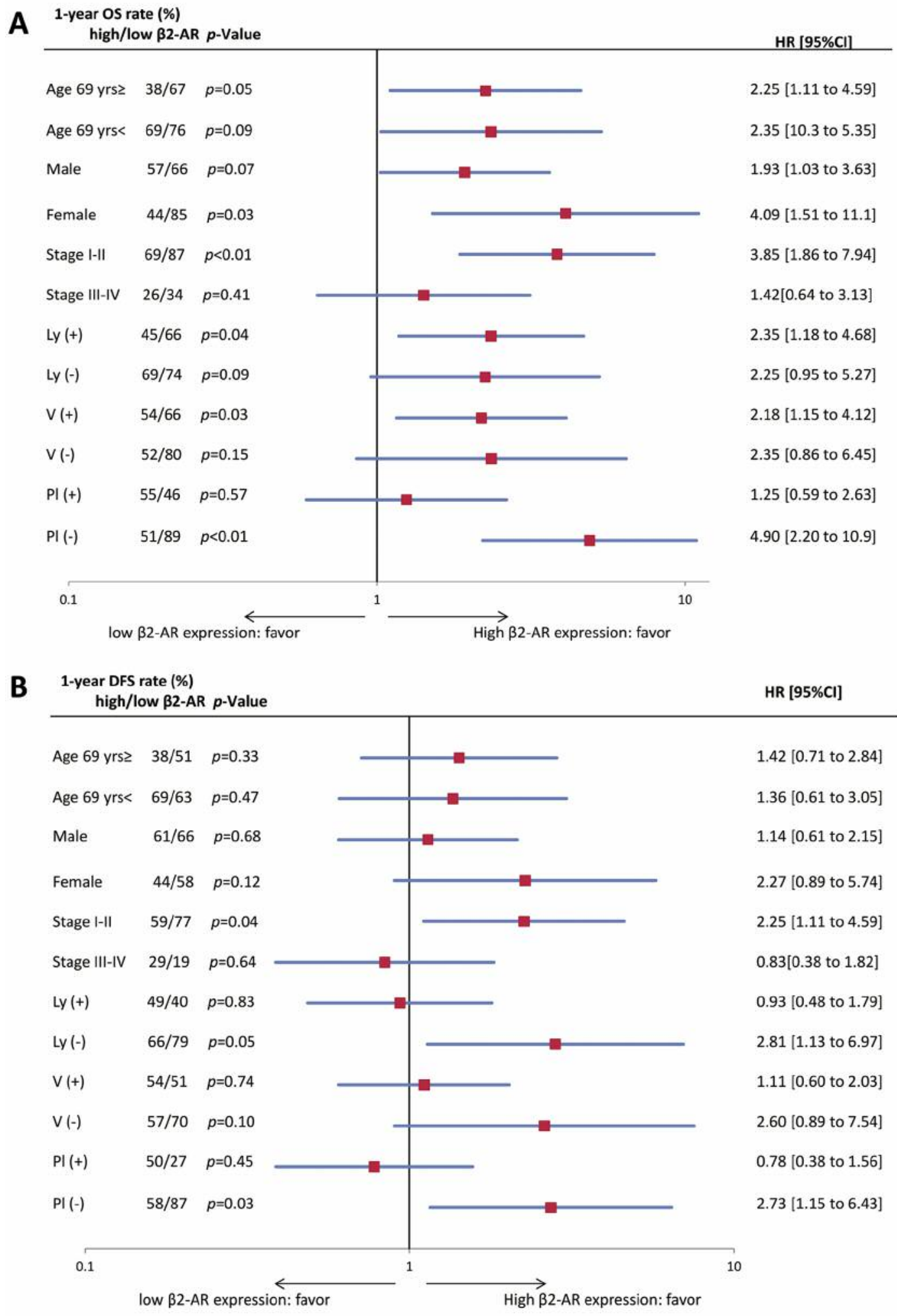

Figure 4. Inlet $A$ shows the forest plot of one-year OS rates according to $\beta 2$-adrenergic receptor $(\beta 2 A R)$ expression for each variable. Inlet $B$ shows the forest plot of one-year DFS rates according to $\beta 2 A R$ expression for each variable. ${ }^{*} p<0.05$ is considered statistically significant, calculated with continuous variable. OS, Overall survival; DFS, disease-free survival; CI, confidence interval; ly, lymphatic permeation; v, vascular invasion; pl, pleural invasion; $\beta 2$-AR, $\beta 2$-adrenergic receptor; HR, hazard ratio. 
Table II. Univariate and multivariate survival analysis in all patients and patients with early-stage disease.

\begin{tabular}{|c|c|c|c|c|c|c|c|c|c|c|}
\hline \multirow[t]{3}{*}{ Variables } & \multicolumn{5}{|c|}{ OS in total patients } & \multicolumn{5}{|c|}{ OS in patients with early stage } \\
\hline & \multicolumn{2}{|c|}{ Univariate analysis } & \multicolumn{3}{|c|}{ Multivariate analysis } & \multicolumn{2}{|c|}{ Univariate analysis } & \multicolumn{3}{|c|}{ Multivariate analysis } \\
\hline & $\begin{array}{c}\text { 1-year rate } \\
(\%)\end{array}$ & $p$-Value & HR & $95 \% \mathrm{CI}$ & $p$-Value & $\begin{array}{c}1-\mathrm{yr} \text { rate } \\
(\%)\end{array}$ & $p$-Value & HR & $95 \% \mathrm{CI}$ & $p$-Value \\
\hline Age $(69 \geq / 69<)$ & $48 / 71$ & 0.46 & & & & $62 / 87$ & 0.54 & & & \\
\hline Gender (female/male) & $50 / 56$ & 0.54 & & & & $74 / 76$ & 0.68 & & & \\
\hline Disease stage (I-II/III-IV) & $74 / 28$ & $<0.01 *$ & 1.84 & 1.41 to 2.41 & $<0.01 *$ & - & - & & & \\
\hline Ly (present/absent) & $51 / 71$ & 0.17 & & & & $68 / 81$ & 0.55 & & & \\
\hline $\mathrm{V}$ (present/absent) & $58 / 61$ & 0.21 & & & & $66 / 95$ & $0.01 *$ & 1.67 & 1.11 to 2.67 & $0.01 *$ \\
\hline $\mathrm{Pl}$ (present/absent) & $52 / 65$ & 0.08 & & & & $74 / 78$ & 0.13 & & & \\
\hline Adjuvant CTx (present/absent) & $66 / 56$ & 0.16 & & & & $72 / 76$ & 0.89 & & & \\
\hline$\beta 2$-AR expression (high/low) & $54 / 71$ & $<0.01 *$ & 1.52 & 1.11 to 2.16 & $<0.01 *$ & $69 / 87$ & $<0.01 *$ & 1.98 & 1.23 to 3.65 & $<0.01 *$ \\
\hline \multirow[t]{2}{*}{ Ki-67 labeling index (high/low) } & $58 / 59$ & 0.85 & & & & $78 / 71$ & 0.88 & & & \\
\hline & \multicolumn{5}{|c|}{ DFS in total patients } & \multicolumn{5}{|c|}{ DFS in patients with early stage } \\
\hline Age $(69 \geq / 69<)$ & $43 / 69$ & 0.15 & & & & $55 / 79$ & 0.20 & & & \\
\hline Gender (female/male) & $58 / 48$ & 0.13 & & & & $69 / 66$ & 0.54 & & & \\
\hline Disease stage (I-II/III-IV) & $67 / 29$ & $<0.01 *$ & 1.75 & 1.32 to 2.16 & $<0.01 *$ & - & - & & & \\
\hline Ly (present/absent) & $46 / 68$ & $0.04 *$ & 1.07 & 0.43 to 1.45 & 0.65 & $58 / 79$ & 0.29 & & & \\
\hline $\mathrm{V}$ (present/absent) & $53 / 54$ & 0.07 & & & & $60 / 77$ & $0.01 *$ & 1.51 & 0.96 to 2.52 & 0.07 \\
\hline Pl (present/absent) & $43 / 67$ & $<0.01 *$ & 1.38 & 1.06 to 1.82 & $0.02 *$ & $53 / 78$ & $<0.01 *$ & 1.38 & 0.94 to 2.11 & 0.09 \\
\hline Adjuvant CTx (present/absent) & $53 / 56$ & 0.91 & & & & $56 / 71$ & 0.48 & & & \\
\hline$\beta 2$-AR expression (high/low) & $54 / 57$ & 0.25 & & & & $59 / 77$ & $0.04 *$ & 1.33 & 0.88 to 2.14 & 0.17 \\
\hline Ki-67 labeling index (high/low) & $51 / 58$ & 0.58 & & & & $66 / 70$ & 0.87 & & & \\
\hline
\end{tabular}

${ }^{*} p<0.05$ is considered statistically significant, calculated with continuous variable. OS, Overall survival; DFS, disease-free survival; CI, confidence interval; Ly, lymphatic permeation; V, vascular invasion; Pl, pleural invasion; $\beta 2$-AR, $\beta 2$-adrenergic receptor; HR, hazard ratio; CTx, chemotherapy.

melanoma $(19,20)$. A clinicopathological study by our group has previously demonstrated high levels of $\beta 2 \mathrm{AR}$ expression in gastric cancer cell lines as compared to normal gastric cells (13). Chisholm et al. demonstrated that $\beta 2 \mathrm{AR}$ is strongly expressed in a variety of vascular tumors $(77 \%$ in angiosarcoma) and thus, speculated that $\beta 2$-antagonists, such as propranolol, could exert their anti-tumor effects (induce apoptosis and reduce the responsiveness to VEGF) in all the $\beta 2$ AR-expressing vascular lesions (21).

To the best of our knowledge, this is the first study to elucidate the association between $\beta 2 \mathrm{AR}$ expression levels and tumor cell aggressiveness in PPC; however, immunohistochemical reports on the clinicopathological significance of $\beta 2 \mathrm{AR}$ expression are still scarce. Further work is necessary to elucidate the mechanism of action of $\beta 2 \mathrm{AR}$ in tumor cells and examine the clinical implications of $\beta 2 A R$ expression levels in various cancer types. In addition, previous studies in pancreatic cancer and PPC have revealed an association between the biological behavior of the tumor and genetic polymorphisms in $\beta 2 \mathrm{AR}$ and EGFR genes, respectively $(22,23)$. Therefore, further investigation of the genomic/genetic markers in PPC is also important to establish the predictive and/or prognostic value of $\beta 2 \mathrm{AR}$ in cancer, and might reveal new therapeutic targets.

The present study has several limitations. First, the number of patients was not sufficient to confirm our results, despite the fact that samples were collected from multiple institutions. This was mainly due to the rarity of the disease; however a larger study should be conducted to validate the results presented here. Second, it is important to examine whether $\beta$-blockers can suppress tumor growth and prolong survival in patients with PPC, since this is not yet known. Finally, further studies are required to investigate whether $\beta 2 \mathrm{AR}$ is associated with the expression of angiogenetic markers, such as VEGF, in PPC, as it is in other cancer types (21).

In conclusion, $\beta 2 \mathrm{AR}$ was highly expressed in patients with surgically-resected PPC and this significantly correlated with lymph node metastasis, lymphatic spread, and tumor cell proliferation. $\beta 2 \mathrm{AR}$ was a significant independent marker for predicting worse prognosis in patients with PPC, especially those with early-stage disease, while it may play an crucial role in the development of tumor aggressiveness and metastases. The inhibition of $\beta 2 \mathrm{AR}$, such as the antagonist propranolol, could be a potential target in patients with PPC. 


\section{Conflicts of Interest}

None of the Authors has any financial or personal relationship with other people or organizations that could inappropriately influence our work.

\section{Acknowledgements}

The Authors thank Drs. Takayuki Kosaka, Toshiki Yajima and Yoichi Ohtaki of Gunma University Hospital for their collection of materials and patients' treatments, Ms. Mai Yagame, Dr. Bolag Altan, and Dr. Erkhem-Ochir Bilguun of Gunma University Hospital for excellent technical assistance.

\section{References}

1 Chang YL, Lee YC, Shih JY and Wu CT: Pulmonary pleomorphic (spindle) cell carcinoma: peculiar clinicopathologic manifestations different from ordinary non-small cell carcinoma. Lung Cancer 34: 91-97, 2001.

2 Travis WD, Brambilla E, Nicholson AG, Yatabe Y, Austin JHM, Beasley MB, Chirieac LR, Dacic S, Duhig E, Flieder DB, Geisinger K, Hirsch FR, Ishikawa Y, Kerr KM, Noguchi M, Pelosi G, Powell CA, Tsao MS and Wistuba I; WHO Panel: The 2015 World Health Organization Classification of Lung Tumors: Impact of Genetic, Clinical and Radiologic Advances Since the 2004 Classification. J Thorac Oncol 10: 1243-1260, 2015.

3 Aronow WS: Current role of beta-blockers in the treatment of hypertension. Expert opinion on Pharmacol 11: 2599-607, 2010.

4 Maudsley S, Pierce KL, Zamah AM, Miller WE, Ahn S, Daaka Y, Lefkowitz RJ and Luttrell LM: The beta(2)-adrenergic receptor mediates extracellular signal-regulated kinase activation via assembly of a multi-receptor complex with the epidermal growth factor receptor. J Biol Chem 275: 9572-9580, 2000.

5 Entschladen F, Drell TL 4th, Lang K, Joseph J and Zaenker KS: Tumour-cell migration, invasion, and metastasis: navigation by neurotransmitters. Lancet Oncol 5: 254-258, 2004.

6 Thaker PH, Han LY, Kamat AA, Arevalo JM, Takahashi R, Lu C, Jennings NB, Armaiz-Pena G, Bankson JA, Ravoori M, Merritt WM, Lin YG, Mangala LS, Kim TJ, Coleman RL, Landen CN, Li Y, Felix E, Sanguino AM, Newman RA, Lloyd M, Gershenson DM, Kundra V, Lopez-Berestein G, Lutgendorf SK, Cole SW and Sood AK: Chronic stress promotes tumor growth and angiogenesis in a mouse model of ovarian carcinoma. Nat Med 12: 939-944, 2006.

7 Ramberg H, Eide T, Krobert KA, Levy FO, Dizeyi N, Bjartell AS, Abrahamsson PA and Taskén KA: Hormonal regulation of beta2-adrenergic receptor level in prostate cancer. Prostate 68 : 1133-1142, 2008.

8 Yang EV, Kim SJ, Donovan EL, Chen M, Gross AC, Webster Marketon JI, Barsky SH and Glaser R: Norepinephrine upregulates VEGF, IL-8, and IL-6 expression in human melanoma tumor cell lines: implications for stress-related enhancement of tumor progression. Brain Behav Immun 23: 267-275, 2009.

9 Shang ZJ, Liu K and Liang DF: Expression of beta2-adrenergic receptor in oral squamous cell carcinoma. J Oral Pathol Med 38: 371-376, 2009.

10 Shi M, Liu D, Duan H, Qian L, Wang L, Niu L, Zhang H, Yong Z, Gong Z, Song L, Yu M, Hu M, Xia Q, Shen B and Guo N: The beta2-adrenergic receptor and Her2 comprise a positive feedback loop in human breast cancer cells. Breast Cancer Res Treat 125: 351-362, 2011.
11 Chen D, Xing W, Hong J, Wang M, Huang Y, Zhu C, Yuan Y and Zeng W: The beta2-adrenergic receptor is a potential prognostic biomarker for human hepatocellular carcinoma after curative resection. Ann Surg Oncol 19: 3556-3565, 2012.

12 Yazawa T, Kaira K, Shimizu A, Shimizu A, Mori K, Nagashima T, Ohtaki Y, Oyama T, Mogi A and Kuwano H: Prognostic significance of $\beta 2$-adrenergic receptor expression in non-small cell lung cancer. Am J Transl Res 8: 5059-5070, 2016.

13 Takahashi K, Kaira K, Shimizu A, Sato T, Takahashi N, Ogawa H, Yoshinari D, Yokobori T, Asao T, Takeyoshi I and Oyama T: Clinical significance of $\beta 2$-adrenergic receptor expression in patients with surgically resected gastric adenocarcinoma. Tumor Biol 37: 13885-13892, 2016.

14 Shimizu A, Kaira K, Mori K, Kato M, Shimizu K, Yasuda M, Takahashi A, Oyama T, Asao T and Ishikawa O: Prognostic significance of $\beta 2$-adrenergic receptor expression in malignant melanoma. Tumor Biol 37: 5971-5978, 2016.

15 Melhem-Bertrandt A, Chavez-Macgregor M, Lei X, Brown EN, Lee RT, Meric-Bernstam F, Sood AK, Conzen SD, Hortobagyi GN and Gonzalez-Angulo AM: Beta-blocker use is associated with improved relapse-free survival in patients with triplenegative breast cancer. J Clin Oncol 29: 2645-2652, 2011.

16 Barron TI, Connolly RM, Sharp L, Bennett K and Visvanathan $\mathrm{K}$ : Beta blockers and breast cancer mortality: a population-based study. J Clin Oncol 29: 2635-2644, 2011.

17 Kaira K, Kawashima O, Endoh H, Imaizumi K, Goto Y, Kamiyoshihara M, Sugano M, Yamamoto R, Osaki T, Tanaka S, Fujita A, Imai H, Kogure Y, Seki Y, Shimizu K, Mogi A, Shitara Y, Oyama T, Kanai Y and Asao T: Expression of amino acid transporter (LAT1 and 4F2hc) in pulmonary pleomorphic carcinoma. Hum Pathol pii: S0046-8177(18)30384-8, 2018. doi: 10.1016/j.humpath.2018.09.020. [Epub ahead of print]

18 Bravo-Calderón DM, Oliveira DT, Marana AN, Nonogaki S, Carvalho AL and Kowalski LP: Prognostic significance of beta2 adrenergic receptor in oral squamous cell carcinoma. Cancer Biomark 10: 51-59, 2012.

19 De Giorgi V, Grazzini M, Gandini S, Benemei S, Lotti T, Marchionni $\mathrm{N}$ and Geppetti P: Treatment with beta-blockers and reduced disease progression in patients with thick melanoma. Arch Intern Med 171: 779-781, 2011.

20 Lemeshow S, Sorensen HT, Phillips G, Yang EV, Antonsen S, Riis AH, Lesinski GB, Jackson R and Glaser R: beta-Blockers and survival among Danish patients with malignant melanoma: a population-based cohort study. Cancer Epidemiol Biomarkers Prev 20: 2273-2279, 2011.

21 Chisholm KM, Chang KW, Truong MT and Kwok S, West RB, Heerema-McKenney AE: $\beta$-adrenergic receptor expression in vascular tumors. Mod Pathol 25: 1446-1451, 2012.

22 Wenjuan Y, Yujun L and Ceng Y: Association of single nucleotide polymorphisms of beta2-adrenergic receptor gene with clinicopathological features of pancreatic carcinoma. Acta histochemica 115: 198-203, 2013.

23 Tamura Y, Fujiwara Y, Yamamoto N, Nokihara H, Horinouchi H, Kanda S, Goto Y, Kubo E, Kitahara S, Tsuruoka K, Tsuta K and Ohe Y: Retrospective analysis of the efficacy of chemotherapy and molecular targeted therapy for advanced pulmonary pleomorphic carcinoma. BMC Res Notes 8: 800, 2015.

Received October 28, 2018

Revised December 12, 2018

Accepted December 13, 2018 